\title{
Changes in body weight among colorectal cancer survivors treated with and without chemotherapy
}

\author{
M. van Zutphen, A. Geelen, E. Kampman and F.J.B. van Duijnhoven for the COLON study \\ Division of Human Nutrition, Wageningen University and Research, The Netherlands.
}

Excess body weight is one of the well-established risk factors for the development of colorectal cancer (CRC) and weight after CRC diagnosis seems to be associated with prognosis and long-term health. Few studies examined weight change after CRC diagnosis. They found that weight gain after diagnosis was common among CRC patients treated with adjuvant chemotherapy ${ }^{(1-3)}$. However, based on these studies we cannot simply conclude that CRC patients gain weight, they could also be regaining weight that was lost before diagnosis. Therefore, the aim of this study was to describe overall weight trajectories from two years before CRC diagnosis to two years post-diagnosis and to see if they differ between those treated with and without adjuvant chemotherapy.

We included 1,182 incident CRC patients who underwent surgery with curative intend (stage I-III) from the prospective COLON cohort study $^{(4)}$. Participants completed a questionnaire shortly after diagnosis on current weight, weight two years before diagnosis and personal characteristics. They also reported on current weight at 6 months, 1 year, and 2 years after diagnosis. Clinical data was collected through the Dutch ColoRectal Audit ${ }^{(5)}$. We used an unadjusted linear mixed model with serial correlation to analyse weight trajectories.

Patients had a mean age of 66 (SD 8.7) years, $64 \%$ were male, $38 \%$ had stage III disease, $67 \%$ had colon cancer and $25 \%$ received adjuvant chemotherapy. In total, mean weight change from diagnosis to 2 years after diagnosis was +1.5 (SE 0.2$) \mathrm{kg}$ with $21 \%$ of patients having $\geq 5 \%$ weight gain and $9 \%$ of patients having $\geq 5 \%$ weight loss. However, when the two years before CRC diagnosis were taken into account, mean weight change was -0.6 (SE 0.2) $\mathrm{kg}$ over the 4 year period. No differences in weight trajectory were detected between treatment groups (Fig. 1; $\mathrm{p}=0.51$ ). Analyses will be continued with more sophisticated linear mixed models. Furthermore, we will assess which personal and/or clinical factors are associated with changes in body weight.

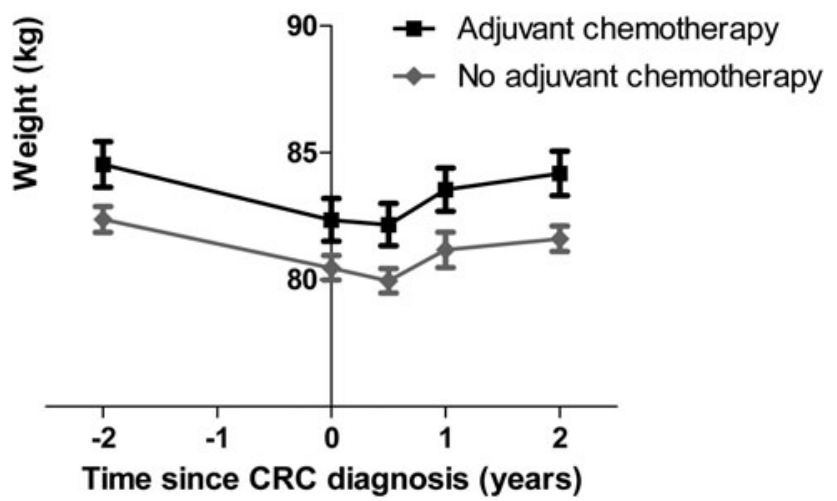

Fig. 1. Weight trajectories in CRC survivors. Data presented as mean $\pm \mathrm{SE}$.

In conclusion, preliminary analyses showed that there was some weight gain in the two years following CRC diagnosis. However, overall weight after CRC diagnosis was not higher than mean weight two years before CRC diagnosis. Furthermore, weight trajectories were similar for patients treated with or without adjuvant chemotherapy.

1. Meyerhardt JA, Niedzwiecki D, Hollis D et al. (2008) J Clin Oncol 26, 4109-4115.

2. Winkels RM, Snetselaar T, Adriaans A et al. (2016) Cancer Treat Res Commun 9, 111-115.

3. Vergidis J, Gresham G, Lim HJ et al. (2016) Clin Colorectal Cancer 15, 16-23.

4. Winkels RM, Heine-Broring RC, Van Zutphen M et al. (2014) BMC Cancer 14, 374.

5. Van Leersum NJ, Snijders HS, Henneman D et al. (2013) Eur J Surg Oncol 39, 1063-1070. 